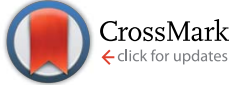

Cite this: RSC Adv., 2016, 6, 2993

\title{
Powering tyrosol antioxidant capacity and osteogenic activity by biocatalytic polymerization $\uparrow$
}

\author{
Stefano Antenucci, ${ }^{\text {ab }}$ Lucia Panzella, ${ }^{* c}$ Hermes Farina, ${ }^{a}$ Marco Aldo Ortenzi, ${ }^{\text {ab }}$ \\ Enrico Caneva, ${ }^{d}$ Simona Martinotti, ${ }^{e}$ Elia Ranzato, ${ }^{e}$ Bruno Burlando, ${ }^{e f}$ Marco d'Ischia, ${ }^{c}$ \\ Alessandra Napolitano ${ }^{c}$ and Luisella Verotta ${ }^{a b}$
}

Oxidative polymerization of tyrosol by horseradish peroxidase (HRP) $-\mathrm{H}_{2} \mathrm{O}_{2}$ afforded an insoluble product (oligotyrosol, OligoTyr) consisting of mixture of linear oligomers (up to 11-mer) with limited benzylic branching points, as evidenced by ESI-MS and solid state ${ }^{13} \mathrm{C}$ NMR analysis. OligoTyr proved to be significantly more active than tyrosol in several antioxidant assays and was not toxic to human osteosarcoma SaOS-2 cells, stimulating alkaline phosphatase (ALP) activity at day 7 in a similar manner as tyrosol. However, when loaded at $5 \% \mathrm{w} / \mathrm{w}$ into highly porous polylactic acid (PLA) scaffolds featuring hierarchical structures, OligoTyr caused a significant increase in the ALP activity of SaOS-2 cells compared to PLA alone, while tyrosol was completely inactive. A release of ca. 5\% from PLA was determined after 1 week in a physiological medium. No significant influence on calcium release from PLA scaffolds containing $5 \% \beta$-tricalcium phosphate was observed.

Received 2nd November 2015 Accepted 20th December 2015

DOI: $10.1039 / \mathrm{c} 5 \mathrm{ra} 23004 \mathrm{~g}$

www.rsc.org/advances processes attract growing interest due to their environmental compatibility associated with the use of catalytic amounts of the enzyme and $\mathrm{H}_{2} \mathrm{O}_{2}$ as an easily available low cost oxidant. The reaction on monophenolic substrates proceeds via one-electron oxidation leading to the generation of phenoxyl radicals intermediates which evolve via sequential oxidative coupling steps. ${ }^{2}$ Examples of biocatalytic synthesis of oligo- and polyphenols include the horseradish peroxidase (HRP)-catalyzed polymerization of phenol, ${ }^{3-5} \mathrm{~m}$-cresol, ${ }^{6}$ bisphenol A, ${ }^{6}$ cardanol, ${ }^{7}$ tyrosine esters, ${ }^{7}$ and guaiacol, ${ }^{8}$ HRP- and soybean peroxidase (SBP)catalyzed oxidative polymerization of $m$-substituted phenol derivatives, ${ }^{9}$ and fungal laccase polymerization of methoxyphenols $^{\mathbf{1 0}}$ (for reviews on enzymatic synthesis of polyphenols, see ref. 11-13).

Although increasing interest is currently being focused on the manifold technological opportunities offered by bioinspired phenolic polymers, e.g. for the preparation of resins and coating $\mathrm{s}^{14-16}$ or for the stabilization of polymers, ${ }^{17,18}$ comparatively less attention has so far been devoted to the use of polymers of natural phenolics as antioxidant additives for biocompatible scaffolds for biomedical applications, e.g. tissue engineering and bone regeneration. Antioxidant compounds have recently been exploited in tissue engineering as promoters of osteoblast differentiation, ${ }^{19}$ since they can counteract the inhibitory effects of reactive oxygen species (ROS) on the process of bone formation by osteoblastic cells. ${ }^{20-23}$ Natural polyphenolic compounds, such as resveratrol and green tea catechins, have been shown to increase alkaline phosphatase (ALP) activity and collagen mineralization by enhancing calcium deposition in mesenchymal stem cells. ${ }^{24,25}$ Olive oil polyphenols such as 
oleuropein and hydroxytyrosol can stimulate the deposition of calcium in osteoblastic MC3T3-E1 cells. ${ }^{26}$

Despite the useful property of natural polyphenols to enhance bone formation, a major drawback is that they are rapidly metabolized and excreted from the body, making controlled release at the site of bone loss an important goal.

Recently, considerable interest has been directed to threedimensional porous scaffolds made of biomaterials such as polycaprolactone (PCL) and polylactic acid (PLA) for antioxidant release in biological tissues. ${ }^{27-31}$ PLA is one of the few biodegradable polymers approved for human use by the FDA that has reached the clinical application stage, ${ }^{32}$ and offers advantages including commercial availability, low cost and easy purification procedures. Moreover, PLA enables implementation of highly porous nanostructures under suitable preparation conditions. ${ }^{33,34}$ A few examples in the literature report preparation of electrospun PLA or PLA/polyethylene oxide fibers incorporating polyphenols like genistein or caffeic acid with improved antioxidant properties for use in wound healing. ${ }^{35,36}$

Accelerated bone regeneration has been reported by supplementing PCL or PLA scaffolds with bioactive molecules like zoledronate $^{37}$ hyaluronic acid, ${ }^{38}$ dexamethasone, ${ }^{39}$ ciprofloxacin, ${ }^{40}$ and polyphenols, ${ }^{25,41}$ that increase proliferation and differentiation of mesenchymal stem cells into osteoblasts and promote osteoblast proliferation and calcium deposition. However, in most cases a major issue is related to the facile and uncontrolled release of low molecular weight phenols from the scaffold into the contact medium. Accordingly, a convenient solution to this issue may derive from the use of phenolic polymers rather than monomers. These polymers would not only exhibit lower tendency to be released from the scaffolds but may display a greater chemical stability and similar or even better antioxidant activity. It has been recently demonstrated in this connection that phlorotannins are good supplemental bioactive agents for enhancing the bone tissue growth of PCL/ $\beta$ tricalcium phosphate ( $\beta$-TCP) composite scaffolds as monomers, being more active in the oligomeric forms. ${ }^{42}$ Although phlorotannins are available in large amounts from brown algae by facile and reproducible isolation protocols, their purification from other polyphenols requires expensive chromatographic treatments. $^{42}$

An attractive candidate for the preparation of all-natural antioxidant phenolic polymers is tyrosol (2-(4-hydroxyphenyl) ethanol). Tyrosol is one of the most representative phenolic constituent of extra virgin olive oil and has been the focus of increasing interest over the past decades because of a range of biological and pharmacological properties, such as antimicrobial, antithrombotic and anti-inflammatory activities. ${ }^{\mathbf{4 3 - 4 5}}$ Recently, high tensile strength, aromatic-aliphatic polycarbonates based on tyrosol and homovanillyl alcohol, that exhibit enzymatic surface erosion by lipase, were reported to support cell attachment and proliferation of human mesenchymal stem cells (hMSCs). ${ }^{46}$ Most of the beneficial properties of tyrosol have been ascribed to its potent antioxidant and scavenging properties against ROS and reactive nitrogen species generated in settings of oxidative stress. ${ }^{43,47,48}$
In this paper we report the first preparation and characterization of a polymer of tyrosol, OligoTyrosol (OligoTyr), via a convenient $\mathrm{HRP} / \mathrm{H}_{2} \mathrm{O}_{2}$-catalyzed oxidation process, and disclose the potential of OligoTyr incorporated into a PLAscaffold for use in bone tissue engineering. Specific aims of this study include: (a) preparation, structural characterization and evaluation of the antioxidant and osteogenic properties of OligoTyr; (b) preparation and characterization of a PLA-OligoTyr composite scaffold at high porosity; (c) evaluation of OligoTyr release kinetics from PLA-OligoTyr scaffold in vitro.

\section{Experimental section}

\subsection{Materials and methods}

All chemicals were purchased from Sigma-Aldrich and were used without any further purification. PLA, trademark Natureworks ${ }^{\circledR} 4043 \mathrm{D}$ (D-isomer content $\approx 4.3 \%$ as declared by the producer) was purchased from Resinex Srl, Italy.

${ }^{1} \mathrm{H}$ NMR spectra were recorded on a Bruker spectrometer. Chemical shifts values were referenced to TMS.

Solid-state ${ }^{13} \mathrm{C}$ cross polarization-magic angle spinning (CPMAS) spectra were collected at $125.77 \mathrm{MHz}$ on a $500 \mathrm{MHz}$ Bruker BioSpin NMR Spectrometer Avance ${ }^{\mathrm{TM}} 500$, operating at a static field of 11.7 tesla and equipped with a $4 \mathrm{~mm}$ MAS probe, spinning the sample at the magic angle, at speeds up to $15 \mathrm{kHz}$ that, with the addition of high power ${ }^{1} \mathrm{H}$ decoupling capability, allows to decrease or eliminate homo and heteronuclear anisotropies. All the samples were prepared by packing them in zirconia $\left(\mathrm{ZrO}_{2}\right)$ rotors, closed with Kel-F caps $(80 \mu \mathrm{L}$ internal volume) and the spinning speed (MAS) was optimized at $12 \mathrm{kHz}$, after some experiments run in the range 4-12 kHz. Crosspolarization (CP) spectra, under Hartmann-Hahn conditions, were recorded with a variable spin-lock sequence (ramp CPMAS), and a relaxation delay of $2 \mathrm{~s}$; a ${ }^{1} \mathrm{H} \pi / 2$ pulse width of $4.0 \mu \mathrm{s}$ was employed. Contact time was varied in the range 1.5$2.5 \mathrm{~ms}$. In some experiments high power proton decoupling was applied during acquisition without cross polarization.

MS spectra were collected on a LCQ Advantage Thermo Fisher Mass Spectrometer equipped with a ESI source (spray voltage: $4.5 \mathrm{kV}$, capillary temperature: $275.90{ }^{\circ} \mathrm{C}$, sheat gas flow rate: 15 arbitrary units; nebulizer pressure: 50 psi, fragmentor voltage: $50 \mathrm{~V}$, gain 1.0). Spectra were obtained from samples dissolved in methanol. MALDI mass spectra were obtained using a Bruker Daltonics Microflex MALDI-TOF apparatus, and $\alpha$-cyano-4-hydroxycinnamic acid as matrix.

UV-vis absorption measurements were performed with a PerkinElmer Lambda 35 UV/VIS or a Jasco V-730 Spectrophotometer.

The molecular weight of acetylated OligoTyr and PLA were determined by gel permeation chromatography (GPC) using a size exclusion chromatography (SEC) system having Waters 1515 Isocratic HPLC pump and a four Shodex GPC KF column set, with a Waters 2487 Dual $\lambda$ Absorbance detector using a flow rate of $1 \mathrm{~mL} \mathrm{~min}^{-1}$ and $20 \mu \mathrm{L}$ as injection volume. The detector was set at $280 \mathrm{~nm}$. Samples were prepared dissolving $5 \mathrm{mg}$ of sample in $1 \mathrm{~mL}$ of anhydrous dichloromethane. $o$-Dichlorobenzene was used as internal standard (retention time $46 \mathrm{~min}$ ). 
Polystyrene samples (450-7600 Da) from American Polymer Standards, Mentor, $\mathrm{OH}$, were used as standard for the determination of OligoTyr molecular weight. For the determination of PLA molecular weight another set of polystyrene samples (9730-1600 $000 \mathrm{Da}$ ) from the same producer was used (number average molecular weight $\left(M_{\mathrm{n}}\right)$ : $90702 \mathrm{Da}$; weight average molecular weight $\left(M_{\mathrm{w}}\right)$ : $171573 \mathrm{Da}$; polydispersity index: 1.89).

Thermogravimetric analysis (TGA) was performed with a PerkinElmer TGA4000 using temperature program (30-600 ${ }^{\circ} \mathrm{C}$ ) at $20{ }^{\circ} \mathrm{C} \mathrm{min}^{-1}$ under a nitrogen flow of $20 \mathrm{~mL} \mathrm{~min}^{-1}$.

Analytical and preparative TLC were carried out on silica gel plates (0.25 and $0.50 \mathrm{~mm}$, respectively).

\subsection{Preparation of OligoTyr}

Tyrosol (400 mg) was dissolved in the minimum quantity of methanol and added to $0.1 \mathrm{M}$ phosphate buffer (pH 6.8) (19 mL) to a final concentration of $150 \mathrm{mM}$. A solution (3 mL) of HRP (188 $\mathrm{U} \mathrm{mL}^{-1}$, final concentration $25 \mathrm{U} \mathrm{mL}^{-1}$ ) (final monomer to enzyme molar ratio $=50000)$ and $30 \%$ hydrogen peroxide $(694$ $\mu \mathrm{L}$, final concentration $300 \mathrm{mM}$ ) were added in two aliquots at 1 $\mathrm{h}$ time intervals. After $2 \mathrm{~h}$ under vigorous stirring the mixture was centrifuged (3000 rpm, 15 minutes), the resulting precipitate was washed three times with water and lyophilized to give a yellowish powder (305 mg, 75\% yield w/w). Acetylated OligoTyr was prepared by treatment of $10 \mathrm{mg}$ of sample with acetic anhydride $(500 \mu \mathrm{L})$ and pyridine $(10 \mu \mathrm{L})$ at room temperature overnight.

\subsection{Isolation of dimer and trimer of tyrosol}

Tyrosol (90 mg) was dissolved in the minimum quantity of methanol and added to $0.1 \mathrm{M}$ phosphate buffer (pH 3.0) (720 $\mathrm{mL}$ ) to a final concentration of $1 \mathrm{mM}$. Ceric ammonium nitrate (726 mg, 2 molar equivalents) was added in two aliquots at $1 \mathrm{~h}$ time intervals. After $2 \mathrm{~h}$ under vigorous stirring the mixture was extracted with ethyl acetate $(3 \times 500 \mathrm{~mL})$ and the combined organic layers were dried over $\mathrm{Na}_{2} \mathrm{SO}_{4}$ and taken to dryness. The residue ( $42 \mathrm{mg}$ ) was treated overnight with acetic anhydride $(1.5 \mathrm{~mL})$ and pyridine $(75 \mu \mathrm{L})$. After removal of the solvents, the residue (59 $\mathrm{mg}$ ) was dissolved in chloroform and fractionated by preparative TLC (eluant cyclohexane/ethyl acetate $1: 1 \mathrm{v} / \mathrm{v})$ to give the acetylated dimer $1\left(R_{\mathrm{f}} 0.59,6 \mathrm{mg}\right.$, $4 \%$ yield $)$ and trimer $2\left(R_{\mathrm{f}} 0.50,6 \mathrm{mg}, 4 \%\right.$ yield $)$ as light yellow oil in pure form.

1. ${ }^{1} \mathrm{H}$ NMR (400 MHz, $\left.\mathrm{CD}_{3} \mathrm{OD}\right): \delta 1.98(\mathrm{~s}, 3 \mathrm{H}), 2.00(\mathrm{~s}, 3 \mathrm{H})$, $2.96(\mathrm{t}, 2 \mathrm{H}, J=6.8 \mathrm{~Hz}), 4.28(\mathrm{t}, 2 \mathrm{H}, J=6.8 \mathrm{~Hz}), 7.10(\mathrm{~d}, 1 \mathrm{H}, J=$ $8.4 \mathrm{~Hz}), 7.14$ (d, $1 \mathrm{H}, J=2.0 \mathrm{~Hz}), 7.29(\mathrm{dd}, 1 \mathrm{H}, J=8.4,2.0 \mathrm{~Hz})$. ESI(+)MS: $m / z 443\left([\mathrm{M}+\mathrm{H}]^{+}\right)$.

2. ${ }^{1} \mathrm{H}$ NMR (400 MHz, $\left.\mathrm{CD}_{3} \mathrm{OD}\right): \delta 2.00(\mathrm{~s}, 9 \mathrm{H}), 2.01(\mathrm{~s}, 3 \mathrm{H})$, $2.04(\mathrm{~s}, 6 \mathrm{H}), 2.96(\mathrm{~m}, 6 \mathrm{H}), 4.27(\mathrm{~m}, 6 \mathrm{H}), 7.11(\mathrm{~d}, 2 \mathrm{H}, J=8.4 \mathrm{~Hz})$, 7.16 (br s, 2H), 7.17 (s, 2H), $7.30(\mathrm{dd}, 2 \mathrm{H}, J=8.4,2.0 \mathrm{~Hz}) . \operatorname{ESI}(+)$ MS: $m / z 663\left([\mathrm{M}+\mathrm{H}]^{+}\right)$.

\subsection{2,2-Diphenyl-1-picrylhydrazyl (DPPH) assay}

The assay was carried out as described, ${ }^{49}$ using a $200 \mu \mathrm{M}$ solution of DPPH and a $0.69 \mathrm{mg} \mathrm{mL}^{-1}$ solution of OligoTyr or tyrosol (both in methanol). Trolox was used as reference compound.
OligoTyr, tyrosol or Trolox $(200 \mu \mathrm{L})$ was added to DPPH $(2 \mathrm{~mL})$ and the absorbance at $515 \mathrm{~nm}$ was determined after $20 \mathrm{~min}$.

\subsection{Ferric reducing/antioxidant power (FRAP) assay}

The assay was carried out as described,,$^{50}$ using a $20 \mathrm{mM}$ solution of $\mathrm{FeCl}_{3} \times 6 \mathrm{H}_{2} \mathrm{O}$ in water, a $10 \mathrm{mM}$ solution of 4,6-tris(2-pyridyl)$s$-triazine (TPTZ) in $40 \mathrm{mM} \mathrm{HCl}$, and a $0.69 \mathrm{mg} \mathrm{mL}^{-1}$ solution of OligoTyr or tyrosol as above. Trolox was used as reference compound. A solution made up of acetate buffer (pH 3.6) (3 mL) plus $\mathrm{Fe}^{3+}$ solution $(300 \mu \mathrm{L})$ and TPTZ solution $(300 \mu \mathrm{L})$ was treated with 10-30 $\mu \mathrm{L}$ of tyrosol, OligoTyr or Trolox solution and after $10 \mathrm{~min}$ the absorbance at $593 \mathrm{~nm}$ was measured.

\subsection{Hydroxyl radical scavenging assay}

The assay ${ }^{51}$ was carried out using the following solutions: 20 $\mathrm{mM} \mathrm{FeCl}_{2} \times 4 \mathrm{H}_{2} \mathrm{O}$ in $40 \mathrm{mM} \mathrm{HCl}, 20 \mathrm{mM} \mathrm{Na}{ }_{2}$ EDTA in water, 50 $\mathrm{mM} \mathrm{H} \mathrm{H}_{2} \mathrm{O}_{2}, 10 \mathrm{mM}$ salicylic acid in $0.2 \mathrm{M}$ phosphate buffer $(\mathrm{pH}$ 7.4), $268 \mathrm{U} \mathrm{mL}^{-1}$ catalase in $0.2 \mathrm{M}$ phosphate buffer ( $\mathrm{pH} 7.4$ ), and $0.69 \mathrm{mg} \mathrm{mL}^{-1}$ OligoTyr or tyrosol as above. The solution of OligoTyr and tyrosol were further diluted in water to a final concentration of $0.3,1,3,7$ or $14 \mu \mathrm{g} \mathrm{mL}^{-1}$. To $1.5 \mathrm{~mL}$ of $0.2 \mathrm{M}$ phosphate buffer (pH 7.4) $500 \mu \mathrm{L}$ of salicylic acid, $250 \mu \mathrm{L}$ of EDTA, $250 \mu \mathrm{L}$ of $\mathrm{Fe}^{2+}, 2 \mathrm{~mL}$ of water, OligoTyr or tyrosol at the desired concentration and $500 \mu \mathrm{L}$ of the $\mathrm{H}_{2} \mathrm{O}_{2}$ solution were added. After $10 \mathrm{~min} 500 \mu \mathrm{L}$ of the catalase solution was added and the mixtures were analyzed by HPLC. Ascorbic acid was used as reference compound. HPLC analysis was carried out on an octadecylsilane column $250 \times 4.60 \mathrm{~mm}(5 \mu \mathrm{m})$ using a gradient based on $10 \mathrm{mM}$ phosphoric acid ( $\mathrm{pH} 2.5$ )/ acetonitrile (from $10 \%$ to $60 \%, 0-35 \mathrm{~min}$ ) as the eluant, at a flow rate of $0.7 \mathrm{~mL} \mathrm{~min}^{-1}$; detection wavelength was set at 280 $\mathrm{nm}$. Percent inhibition was determined by quantitative analysis of dihydroxybenzoic acids in the presence and in the absence of the antioxidant (OligoTyr, tyrosol or ascorbic acid).

\subsection{Preparation of PLA scaffolds}

All scaffolds were prepared using PLA after purification. PLA was dissolved in dichloromethane and then re-precipitated in methanol to remove processing additives e.g. antioxidants. Scaffolds were prepared according to two different methods:

Method A. PLA $\left(0.1 \mathrm{mg} \mathrm{mL}^{-1}\right)$ and OligoTyr ( $5 \% \mathrm{w} / \mathrm{w}$ on PLA) were dissolved in THF in a $100 \mathrm{~mL}$ glass flask. Methanol was added at room temperature under mechanical stirring up to 95/ $5 \mathrm{v} / \mathrm{v}$ THF/methanol ratio. The solution was frozen by immersion of the flask into liquid nitrogen and was then poured in warm water. The solid PLA scaffolds that separated were recovered after removal of the solvent by filtration.

Method B. PLA ( $\left.0.1 \mathrm{mg} \mathrm{mL}^{-1}\right)$ and tyrosol or OligoTyr $(5 \% \mathrm{w} /$ $\mathrm{w}$ on PLA) were dispersed in 1,4-dioxane in a $100 \mathrm{~mL}$ glass flask at room temperature and taken under mechanical stirring overnight. The resulting homogeneous dispersion was frozen by immersion of the flask into liquid nitrogen and the solvent was removed by sublimation at room temperature under vacuum $\left(2.5 \times 10^{-3}\right.$ bar $)$. In other experiments PLA scaffolds containing $\beta$-tricalcium phosphate ( $\beta$-TCP) $(5 \% \mathrm{w} / \mathrm{w}$ on PLA, porosimetry $d_{50}=100 \mathrm{~nm}$ ) with or without OligoTyr were prepared. 


\section{$2.8 \mathrm{UV} / \mathrm{VIS}$ analyses}

For determination of the content of OligoTyr in PLA/OligoTyr scaffolds, these latters (3 mg) were dissolved in dichloromethane-methanol $1: 1 \mathrm{v} / \mathrm{v}(3 \mathrm{~mL})$ and the absorbance at 286 $\mathrm{nm}$ was determined. The content of OligoTyr was estimated against a calibration curve built using solutions of OligoTyr in the same solvent.

For $\mathrm{p} K_{\mathrm{a}}$ determination, a $0.6 \mathrm{mg} \mathrm{mL}^{-1}$ solution of tyrosol or OligoTyr in DMSO was diluted 20 times in $0.1 \mathrm{M}$ phosphate buffer at different pHs in the range pH 5-12 and the UV-vis spectrum was recorded.

\subsection{Porosity and swelling measurements}

The porosity of the PLA scaffold prepared according to method $\mathrm{B}$ was determined using two different gravimetric methods. In the first one, related porosity was calculated by eqn (1) using the density of the dry scaffold $\left(\rho_{\mathrm{s}}\right)$ and the density of raw PLA $\left(\rho_{\text {PLA }}\right)$, determined as the ratio between the dry mass and the volume of the scaffolds:

$$
\text { Porosity }(\%)=\left[1-\left(\rho_{\mathrm{s}} / \rho_{\mathrm{PLA}}\right)\right] \times 100
$$

The dry weight of the scaffolds was determined using a high precision balance CPA225D Sartorius, the volume was evaluated by geometrical calculation using a caliper.

In the second method the porosity was evaluated using eqn (2):

$$
\text { Porosity }(\%)=\left\{\left(m_{\mathrm{w}}-m_{\mathrm{D}}\right) /\left[\left(m_{\mathrm{D}} / \rho_{\mathrm{PLA}}\right)+m_{\mathrm{w}}\right]\right\} \times 100
$$

where $m_{\mathrm{w}}$ is the wet weight of the scaffold (contact time between scaffold and water: $96 \mathrm{~h}$ ) and $m_{\mathrm{D}}$ is the dry mass. In this case, the porosity was evaluated as the total amount of water absorbed by the scaffold. Swelling of the wet sample was also evaluated using eqn (3):

$$
\text { Swelling }(\%)=\left[\left(m_{\mathrm{w}}-m_{\mathrm{D}}\right) / m_{\mathrm{D}}\right] \times 100
$$

\subsection{Cell culture and in vitro assays}

In vitro experiments were carried out using the human osteosarcoma cell line SaOS-2 (American Type Culture Collection, Rockville, Md-USA). Cells were grown and subjected to the calcein-AM assay for cell viability after $24 \mathrm{~h} .{ }^{52}$ Briefly, cells were settled overnight in 96-well plates, incubated with variable amounts of the materials, and read in an Infinite 200 Pro plate reader (Tecan, Wien, Austria), at $485 \mathrm{~nm} \lambda_{\text {exc }} / 535 \mathrm{~nm} \lambda_{\text {em }}$. Finely ground materials were used in order to obtain suspensions at different nominal concentrations.

Alkaline phosphatase (ALP) assay was carried out as previously reported. ${ }^{53}$ Cells were exposed to tyrosol or OligoTyr dispersed in water at $5 \mu \mathrm{g} \mathrm{mL} \mathrm{m}^{-1}$ or alternatively to PLA scaffolds using the materials pulverized in liquid nitrogen $(25 \mathrm{mg})$ and dispersed on the bottom of the well in 96-well plates, and grown for 7 days. At the end of the experiments, cells were incubated with $p$-nitrophenol phosphate for $10 \mathrm{~min}$, quenched with $0.5 \mathrm{~N}$
$\mathrm{NaOH}$ solution, and thereafter, the concentration of $p$-nitrophenol was determined with a plate reader at $405 \mathrm{~nm}$. Data were analysed with the $\mathrm{R}$ package, version 3.0.1 (http://www.rproject.org/foundation/). Values of IC50 and 95\% confidence intervals were calculated using a downhill logistic curve.

\subsection{Analysis of OligoTyr release from PLA scaffolds}

Preweighted PLA scaffolds were incubated in phosphatebuffered saline (PBS) at $30 \mathrm{mg} \mathrm{mL}^{-1}$ under static conditions at $37^{\circ} \mathrm{C}$. The absorbance of released OligoTyr was read at 7 day intervals at $286 \mathrm{~nm}$. The concentration of the released material was obtained from standard curves built using OligoTyr solutions of known concentrations and calculated on the basis of the amount of compound loaded in the PLA scaffold. The release was followed over a 28 days period with refreshing of the medium every 7 days. Experiments were run in triplicate.

\subsection{Analysis of calcium release from PLA scaffolds containing $\beta$-TCP}

Preweighted PLA scaffolds were incubated in PBS at $30 \mathrm{mg}$ $\mathrm{mL}^{-1}$ under static conditions at $37^{\circ} \mathrm{C}$ as above. The amount of calcium released was determined using the $o$-cresolphthalein complexone (CPC) method. ${ }^{54}$ Briefly, the working solution was prepared by dissolving $5 \mathrm{mg}$ of CPC and $100 \mathrm{mg}$ of quinolin-8ol in distilled water, to which a few drops of concentrated hydrochloric acid were added. The $\mathrm{pH}$ was then adjusted to 4.0 with sodium hydroxide, and the solution was diluted to $200 \mathrm{~mL}$ with distilled water. Aliquots of the PBS solution were periodically withdrawn and added to the working solution, and the absorbance at $580 \mathrm{~nm}$ was measured. The concentration of the calcium released was obtained from standard curves built using calcium solutions of known concentrations. The percentage released was calculated based on the initial amount of calcium loaded into the scaffold. Experiments were run in triplicate.

\subsection{Evaluation of PLA crystallinity}

The crystalline content of PLA was evaluated by differential scanning calorimetry (DSC). DSC analyses were conducted on PLA purified as described in Section 2.7 under a nitrogen flow using a Mettler Toledo DSC 1 instrument. The sample was first heated from $25{ }^{\circ} \mathrm{C}$ to $200{ }^{\circ} \mathrm{C}$ at $10{ }^{\circ} \mathrm{C} \mathrm{min}{ }^{-1}$, left for $5 \mathrm{~min}$ at $200{ }^{\circ} \mathrm{C}$ to remove possible residual solvent present after purification, then cooled from $200{ }^{\circ} \mathrm{C}$ to $25^{\circ} \mathrm{C}$ at $-10^{\circ} \mathrm{C} \mathrm{min}{ }^{-1}$, left for $2 \mathrm{~min}$ at $25^{\circ} \mathrm{C}$ and then subjected to a second thermal cycle as above. The crystalline weight fraction $\left(X_{\mathrm{c}}\right)$ of the sample was determined as previously described ${ }^{55}$ according to eqn (4):

$$
X_{\mathrm{c}}=\left[\left(\Delta H_{\mathrm{m}}-\Delta H_{\mathrm{cc}}\right) / \Delta H_{\mathrm{m}}^{\mathrm{o}}\right] \times 100
$$

where $\Delta H_{\mathrm{m}}$ is the heat of fusion measured on the second heating, $\Delta H_{\mathrm{cc}}$ is the cold crystallization heat and $\Delta H_{\mathrm{m}}^{\mathrm{o}}$ is the melting enthalpy of the $100 \%$ crystalline polymeric matrix (93 J $\left.\mathrm{g}^{-1}\right){ }^{56}$ A crystalline fraction of $0.4 \%$ was determined. 


\section{Results and discussion}

\subsection{Synthesis and structural characterization of OligoTyr}

OligoTyr was prepared by $\mathrm{HRP} / \mathrm{H}_{2} \mathrm{O}_{2}$ oxidation of $150 \mathrm{mM}$ tyrosol in phosphate buffer, $\mathrm{pH}$ 6.8. The polymer was obtained in $c a$. $75 \%$ yield on a weight basis as a yellowish solid, fairly soluble in methanol, fully soluble in THF and DMSO, and fairly insoluble in water. It showed a broad absorption maximum centered at $286 \mathrm{~nm}$ (mass extinction coefficient $=38.9 \mathrm{~L} \mathrm{~g}^{-1}$ $\mathrm{cm}^{-1}$ ) and a shoulder at $325 \mathrm{~nm}$ in methanol.

Thermogravimetric analysis (TGA) showed a substantial thermal stability of the material up to $250{ }^{\circ} \mathrm{C}$ (Fig. S1 $\dagger$ ).

To determine the average molecular weight, OligoTyr was subjected to acetylation and the resulting material analyzed by non-aqueous size exclusion chromatography (SEC): a weightaverage molecular weight $\left(M_{\mathrm{w}}\right)$ of 2861 Da and a polydispersity index of 1.28 was found, indicating a homogeneous distribution of the oligomer species (Fig. S2 $\dagger$ ).

ESI(-)MS analysis (Fig. S3†) showed well-detectable clusters of peaks in the mass range up to $2000 \mathrm{~m} / \mathrm{z}$, suggesting the presence of significant levels of oligomers up to at least the undecamer stage, peaking at the heptamer. High batch-to-batch reproducibility in the oligomer distribution was observed. Analysis of the clusters of the pseudomolecular ion peaks [M $\mathrm{H}]^{-}$revealed a repetitive unit of $136 \mathrm{Da}$ which by comparison with the molecular weight of tyrosol of 138 Da suggested a linear polymerization mode typical of monophenols. Similar distribution of oligomers was also observed by MALDI analysis (Fig. S4 $\dagger$ ).

Other valuable structural information was obtained by solid state ${ }^{13} \mathrm{C}$ NMR run in the cross polarization-magic angle spinning (CP-MAS) modality. Preliminarily, the spectrum of tyrosol was run under the same modality (Fig. S5 $\dagger$ ). In addition to the signals around 38 and $63 \mathrm{ppm}$ due to the hydroxyethyl chain, signals around 115 (C-3, C-5), 130 (C-1, C-2, C-6) and 153 (C-4) ppm were well apparent in close analogy to the ${ }^{13} \mathrm{C}$ spectrum run in solution. The CP-MAS spectrum of OligoTyr (Fig. 1) showed intense signals corresponding to the hydroxyethyl chain around 38 and $63 \mathrm{ppm}$, indicating that this moiety is not

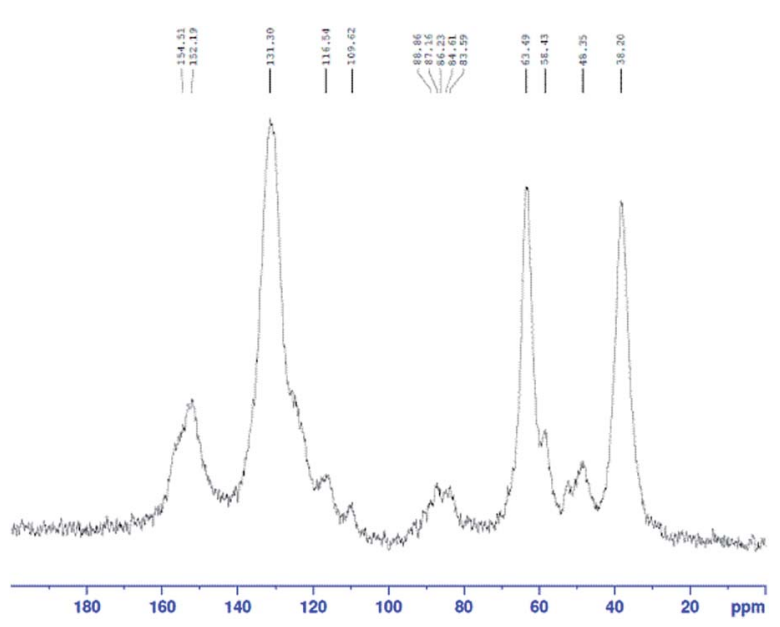

Fig. $1{ }^{13} \mathrm{C}$ CP-MAS NMR spectrum of OligoTyr. significantly involved in the polymerization reaction, although less intense resonances at 48 and 86 ppm were also present. Intense broad signals at around 131 and $152 \mathrm{ppm}$ were well apparent whereas the 115 ppm signal was significantly abated suggesting that the 3 and 5 positions of tyrosol were involved in the coupling.

In order to obtain further information on the mode of polymerization of tyrosol and on the structure of the oligomeric constituents of OligoTyr, other experiments were directed to the isolation of the oligomeric products formed in the early stages of the oxidation of tyrosol. However, no chromatographically defined compounds could be detected in the $\mathrm{HRP} / \mathrm{H}_{2} \mathrm{O}_{2}$ reaction under a variety of conditions.

After several attempts, a procedure was eventually developed, involving $1 \mathrm{mM}$ oxidation of tyrosol with 2 molar equivalents of ceric ammonium nitrate in $0.1 \mathrm{M}$ phosphate buffer followed by extraction with ethyl acetate and acetylation with $\mathrm{Ac}_{2} \mathrm{O}$ /pyridine at room temperature. TLC analysis of the mixture thus obtained showed the presence of two main species eluted at $R_{\mathrm{f}} 0.59$ and 0.50 (eluent cyclohexane/ethyl acetate $1: 1$ ) which could be isolated in pure form from a large scale reaction and subjected to spectral characterization. The ${ }^{1} \mathrm{H}$ NMR spectrum of the $R_{\mathrm{f}}$ 0.59 product displayed an ABX system $(7.10, \mathrm{~d}, J=8.4 \mathrm{~Hz} ; 7.14$, $\mathrm{d}, J=2.0 \mathrm{~Hz}, 7.29, \mathrm{dd}, J=8.4,2.0 \mathrm{~Hz}$ ) typical for a 2,4 -substitued phenol. In addition, two $2 \mathrm{H}$ triplets at $\delta 4.28$ and 2.96 and two singlets for acetyl groups were distinguishable in the high field region. Based on these and ESI(+)MS data (pseudomolecular ion peak $[\mathrm{M}+\mathrm{H}]^{+}$at $\left.m / z 443\right)$ the product was formulated as the acetylated dimer $\mathbf{1}$.<smiles>[R]CCc1ccc([R])c(-c2cc(CCO[R])ccc2[R])c1</smiles>

The ESI(+)MS spectrum of the product at $R_{\mathrm{f}} 0.50$ exhibited a pseudomolecular ion peak $[\mathrm{M}+\mathrm{H}]^{+}$at $\mathrm{m} / \mathrm{z} 663$, indicating a fully acetylated trimer of tyrosol. Consistent with this conclusion was the ${ }^{1} \mathrm{H}$ NMR spectrum showing an $\mathrm{ABX}$ system and a singlet at $\delta 7.17$ in the low field region. The compound was therefore identified as the acetylated trimer 2 .

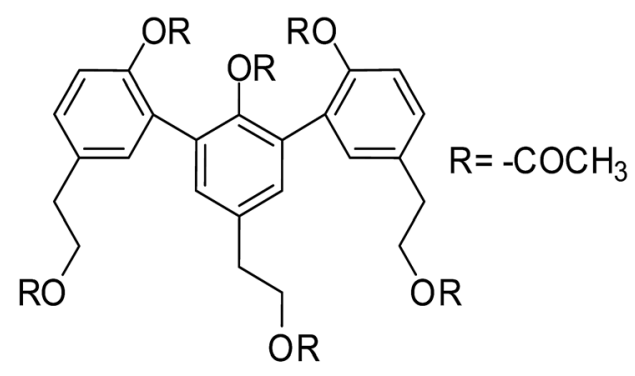

2 
Based on these results and on the ESI(-)MS and NMR data, the representative structure shown in Fig. 2 was proposed for OligoTyr, which was formulated as a mixture of oligomers deriving from a linear polymerization mode with some branching points likely accounting for the observed resonances at 48 and $86 \mathrm{ppm}$ in the CP-MAS spectrum.

As previously reported for dityrosine,${ }^{57}$ a lowering of the $\mathrm{p} K_{\mathrm{a}}$ value from 10.3 for tyrosol to 7.1 for OligoTyr, as determined by UV-vis analysis of the spectra recorded at different pHs (see Fig. S6†), was observed. This suggests that dissociation at the phenol site of OligoTyr is favored over tyrosol due to resonance stabilization of the resulting anion in good agreement with the structure proposed for OligoTyr.

\subsection{Antioxidant properties}

The antioxidant properties of OligoTyr were investigated with respect to tyrosol and reference compounds using commonly used assays such as: (a) the 2,2-diphenyl-1-picrylhydrazyl (DPPH) assay, which determines the H-donor capacity of the antioxidant as quencher of the stable DPPH free radical; ${ }^{49}$ (b) the ferric reducing/antioxidant power (FRAP) assay, which measures the ability of the antioxidant to reduce a $\mathrm{Fe}^{3+}$-tripyridyltriazine complex to the $\mathrm{Fe}^{2+}$ form $;^{50}$ (c) the salicylate assay, which determines the ability of the compound to act as $\mathrm{OH}$ radical scavenger by inhibiting hydroxylation of salicylic acid by the Fenton reagent to give 2,3- and 2,5-dihydroxybenzoic acids (HPLC quantitation). ${ }^{51}$ In the DDPH assay, OligoTyr was found to exert a modest but significant antioxidant effect as compared to trolox ( $97 \pm 1 \%$ reduction), superior to that of the parent compound tyrosol (Table 1). At a final concentration range of 2$30 \mu \mathrm{g} \mathrm{mL} \mathrm{m}^{-1}$, OligoTyr exhibited a 1.3-fold higher reducing capacity toward $1.7 \mathrm{mM} \mathrm{FeCl}_{3}$ in $0.3 \mathrm{M}$ acetate buffer ( $\mathrm{pH}$ 3.6) in comparison with tyrosol. In the $\mathrm{OH}$ radical scavenging salicylate assay OligoTyr proved to be more efficient than ascorbic acid (IC50 $0.81 \pm 0.02 \mu \mathrm{g} \mathrm{mL}^{-1}$ ) in inhibiting salicylate hydroxylation.

From the above set of experiments it could be concluded that OligoTyr has an antioxidant capacity superior to that of the parent compound tyrosol, likely due to the stabilizing effect of

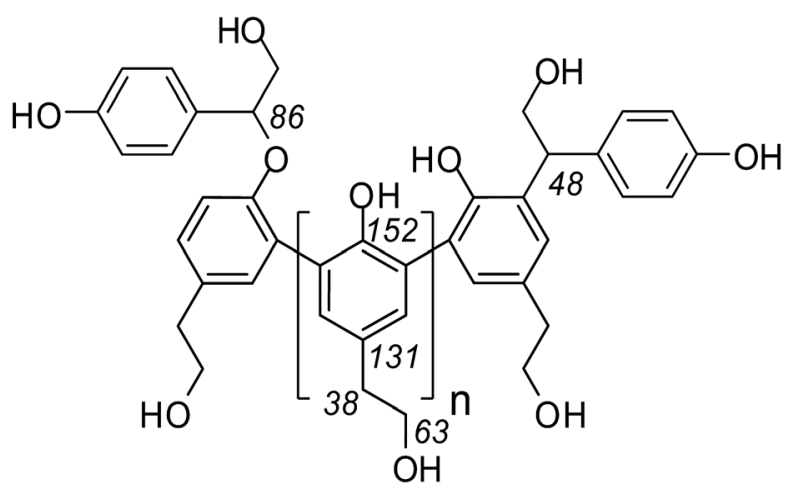

Fig. 2 Proposed representative structures of OligoTyr oligomers responsible for the main peaks of the ESI(-)MS and CP-MAS spectrum. The order of the structural units is arbitrary. Shown are the main carbon resonances observed in the CP-MAS spectrum.
Table 1 Antioxidant properties of tyrosol and OligoTyr

\begin{tabular}{llll}
\hline Sample & $\begin{array}{l}\text { DPPH } \\
\text { reduction }^{a}(\%)\end{array}$ & $\begin{array}{l}\text { Trolox eqs } \\
\text { (FRAP assay) }\end{array}$ & $\begin{array}{l}\text { OH radical scavenging } \\
\text { assay }\left(\text { IC50, } \mu \mathrm{mL}^{-1} \text { ) }\right.\end{array}$ \\
\hline Tyrosol & $24 \pm 1$ & $0.054 \pm 0.002$ & $0.73 \pm 0.01$ \\
OligoTyr & $48 \pm 2$ & $0.072 \pm 0.002$ & $1.16 \pm 0.02$ \\
${ }^{a}$ Calculated at a $0.06 \mathrm{mg} \mathrm{mL}^{-1}$ sample concentration.
\end{tabular}

the aromatic rings in ortho position to the phenoxyl radical in the oligomeric structures.

\subsection{Biocompatibility and osteogenic properties}

The biocompatibility of OligoTyr was preliminarily assessed in comparison to tyrosol by determining the calcein-AM cell viability on human osteosarcoma cell line SaOS-2 at $24 \mathrm{~h}^{52}$ The value obtained (IC50 $>500 \mu \mathrm{g} \mathrm{mL}^{-1}$ ) indicated a very low cytotoxicity of OligoTyr as well as of tyrosol.

As a further experiment, the induction of alkaline phosphatase (ALP) activity, an early marker of osteoblastic differentiation, was evaluated. ${ }^{53}$ Fig. 3 shows that ALP activity was significantly higher in cells grown for 7 days in the presence of tyrosol and OligoTyr, with respect to untreated cells.

Taken together, these results demonstrated more potent antioxidant properties of OligoTyr with respect to tyrosol with comparable biological activity. Accordingly, in further experiments the bioactivity of OligoTyr was investigated in comparison with tyrosol following encapsulation into PLA scaffolds. Prior to biological experiments, the effects of OligoTyr-loading on the morphological properties of PLA scaffolds were investigated.

\subsection{Preparation and characterization of OligoTyr loaded PLA scaffolds}

PLA scaffolds were initially prepared by dissolving PLA and OligoTyr (this latter at $5 \% \mathrm{w} / \mathrm{w}$ loading) in $\mathrm{THF} / \mathrm{methanol}$ at

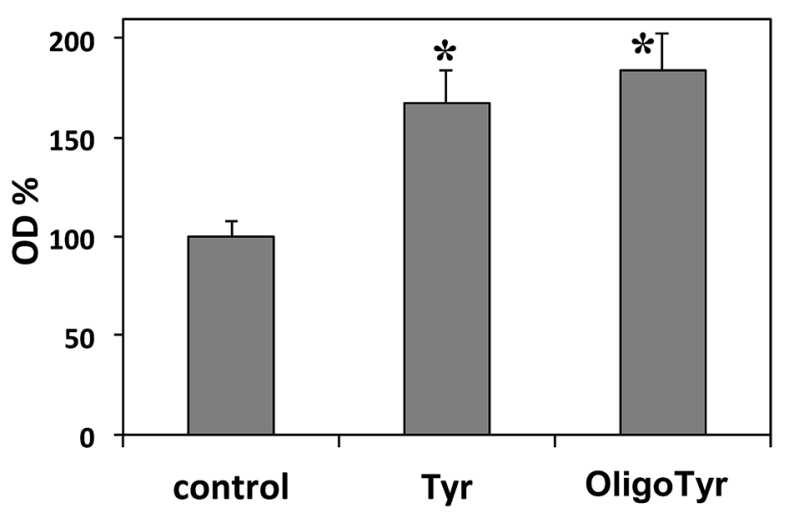

Fig. 3 ALP activities measured in the incubation medium of SaOS-2 cells after 7 days growth in the presence of tyrosol (Tyr) and OligoTyr $\left(5 \mu \mathrm{g} \mathrm{mL}{ }^{-1}\right)$. Data are expressed as mean $\pm \mathrm{SD}(n=8-16)$ of $p-$ nitrophenol optical density (OD) at $405 \mathrm{~nm}$, standardized as percent of control. $* p<0.01$ with respect to control, according to multiple $t$ test with Bonferroni correction. 
$95: 5 \mathrm{v} / \mathrm{v}$ ratio, freezing the solution and then recovering the sponge-like PLA material that separated after pouring the frozen mass into warm water. The OligoTyr loaded PLA scaffolds thus obtained were analyzed by scanning electron microscopy (SEM) in comparison with pure PLA (Fig. S7 $\dagger$ ).

Both materials were porous and showed a "network" structure, but with some differences in the morphology. In particular, in the OligoTyr loaded scaffold small crystals were apparent due likely to OligoTyr remained undissolved in the PLA matrix (see magnification of the images in Fig. S7†). The amount of OligoTyr actually loaded in the scaffold was evaluated by UV/vis spectrophotometry. OligoTyr concentration was found to vary significantly within the scaffold depending on the sampling site, and in all cases was lower (4-4.5\%) than the theoretical one based on a $5 \% \mathrm{w} / \mathrm{w}$ loading. This indicated that the dispersion of OligoTyr in PLA was non-homogenous and that some leakage of OligoTyr occurred during scaffolds preparation. On this basis this method (method A) was judged not reliable and a different protocol (method B) was developed. In order to ensure a satisfactory solubilization of OligoTyr into PLA, 1,4-dioxane was chosen as the solvent. As a first advantage it shows a sublimation temperature $(284.1 \mathrm{~K})^{58}$ far higher than that of THF (164.8 K), ${ }^{59}$ allowing for a convenient removal of the solvent from the bulk material under reduced pressure. In addition, dioxane has already been reported to afford PLA hierarchical scaffolds. ${ }^{60-62}$ Method B involved complete solubilization of either PLA and OligoTyr in dioxane followed by freezing in liquid nitrogen and removal of the solvent by sublimation at room temperature under vacuum. A 5\% loading could be easily obtained under these conditions. For comparison PLA composites containing tyrosol at 5\% loading were also prepared but the materials were not fully characterized nor further investigated as induction of ALP activity on osteosarcoma cell lines (see below) clearly showed the superior properties of OligoTyr.

Fig. 4 shows SEM images of scaffolds obtained from PLA + $5 \% \mathrm{w} / \mathrm{w}$ OligoTyr compared with pure PLA. No OligoTyr crystals were visible even at the higher magnification in the PLA scaffolds obtained by this method. Given the preparation protocol, the content of OligoTyr in the scaffold was assumed to be identical to the theoretical loading.

The porosity of the PLA scaffolds prepared according to method B was determined using two different gravimetric methods. In the first one, related porosity was calculated by eqn (1) using the density of the dry scaffold $\left(\rho_{\mathrm{s}}\right)$ and the density of raw PLA $\left(\rho_{\text {PLA }}\right)$, determined as the ratio between the dry mass and the volume of the scaffolds:

$$
\text { Porosity }(\%)=\left[1-\left(\rho_{\mathrm{s}} / \rho_{\mathrm{PLA}}\right)\right] \times 100
$$

In the second method the porosity was evaluated using eqn (2):

$$
\text { Porosity }(\%)=\left\{\left(m_{\mathrm{w}}-m_{\mathrm{D}}\right) /\left[\left(m_{\mathrm{D}} / \rho_{\mathrm{PLA}}\right)+m_{\mathrm{w}}\right]\right\} \times 100
$$

where $m_{\mathrm{w}}$ is the wet weight of the scaffold after $96 \mathrm{~h}$ contact with water and $m_{\mathrm{D}}$ is the dry mass. In this case, the porosity is
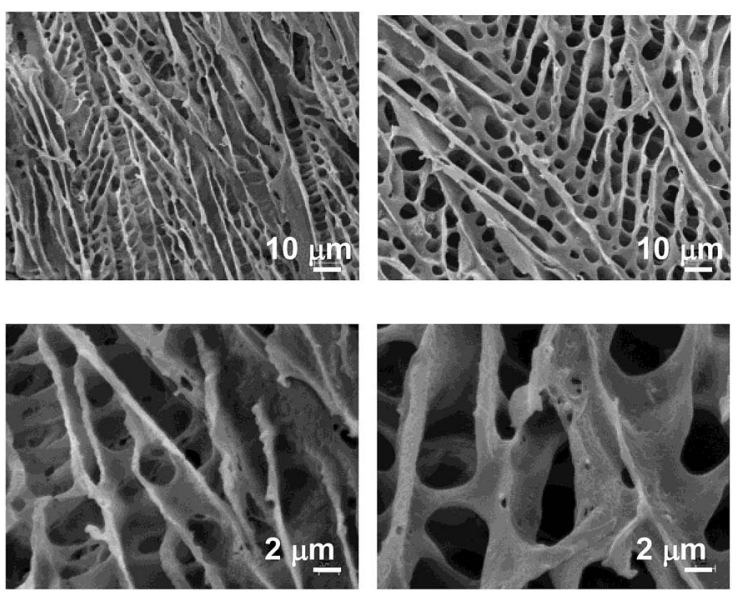

Fig. 4 Top: SEM micrographs of scaffolds obtained from pure PLA (left) and PLA + 5\% OligoTyr (right). Bottom: close-up of the scaffolds shown in the upper row.

evaluated as the total amount of water absorbed by the scaffold. Thus, this latter method allows to estimate the internal connections of the pores inside the scaffold.

A porosity of $86.7 \%$ was calculated by eqn (1) whereas the value obtained using eqn (2) was $54.5 \%$. Such a difference can be explained considering the reduced accessibility of the internal cells of the scaffold, combined with the low wettability of PLA that, based on its static water contact angle $\left(65^{\circ}\right),{ }^{63}$ is halfway between highly hydrophilic (contact angle $0^{\circ}$ ) and hydrophobic (contact angle $>90^{\circ}$ ) materials. Porosity and swelling measurements were repeated on PLA scaffolds over a period of $120 \mathrm{~h}$. Results are presented in Table 2, indicating that the kinetic of water absorption is low, reaching a plateau only after $96 \mathrm{~h}$. These properties are not likely modified by OligoTyr given the low loading chosen. It is not expected that OligoTyr affects the wettability of the scaffold since it is fairly insoluble in water; furthermore the scaffolds with and without OligoTyr addition have closely similar structures with the same average pore size.

\subsection{Osteogenic properties of OligoTyr/PLA and tyrosol/PLA scaffolds}

To assess the potential of OligoTyr/PLA and tyrosol/PLA scaffolds as osteogenic promoters, cells were grown for 7 days on the composites finely ground. Data in Fig. 5 show that 5\% OligoTyr caused a small but significant increase in the ALP activity compared to PLA alone, while 5\% tyrosol was completely inactive.

To gain an insight into the effect of $5 \%$ OligoTyr on ALP stimulation by PLA, the release of OligoTyr from the PLA scaffolds and the effects on calcium release were next examined.

\subsection{Release of OligoTyr from PLA scaffolds}

The rate of release of OligoTyr from PLA scaffolds at 5\% loading was monitored at $37^{\circ} \mathrm{C}$ in physiological medium (phosphatebuffered saline, PBS) by UV spectrophotometry. A 5.2\% release was observed after 7 days incubation, while a cumulative $10.3 \%$ 
Table 2 Porosity and swelling properties of PLA scaffold in contact with water at different times

\begin{tabular}{|c|c|c|c|c|}
\hline & \multicolumn{4}{|c|}{ Time (h) } \\
\hline & 2 & 24 & 96 & 120 \\
\hline Porosity $^{a}(\%)$ & 37.1 & 46.7 & 54.7 & 54.5 \\
\hline Swelling (\%) & 59.0 & 87.6 & 120.8 & 119.8 \\
\hline
\end{tabular}

release was observed after 4 weeks incubation (Fig. S8†). From these data it appears that, though a quite limited amount of OligoTyr is released over the timespan of the ALP activity assay (7 days), an appreciable ALP activity stimulation is observed.

The controlled and prolonged release from PLA scaffolds is likely the result of the entrapment of the phenolic polymer into PLA, which acts as a barrier for the release in PBS. This is in line with the reduced accessibility of the internal cells of the scaffold indicated by the swelling and porosity properties of the material (Table 1). These results confirm that encapsulation of OligoTyr into a PLA scaffold is a convenient means of achieving controlled release of the bioactive phenolic polymer into the biological tissue, a desirable property for therapeutic uses. ${ }^{25}$

\subsection{Effect of OligoTyr on calcium release from PLA scaffolds}

In the last series of experiments the effect of OligoTyr on the release of calcium from PLA scaffolds was investigated. $\beta$-Tricalcium phosphate ( $\beta$-TCP) was chosen to prepare the composite since this biomaterial has been widely used in view of its osteoconductive properties and cellular activities for regenerating bone tissues. ${ }^{42,64}$ PLA scaffolds containing $5 \% \mathrm{w} / \mathrm{w}$ $\beta$-TCP with or without $5 \%$ OligoTyr loading were investigated. As shown in Fig. 6, the composite scaffold showed a linear, slow release of the cation, reaching a ca. $70 \%$ value after 4 weeks. Interestingly, compared to PLA alone, it could be concluded that

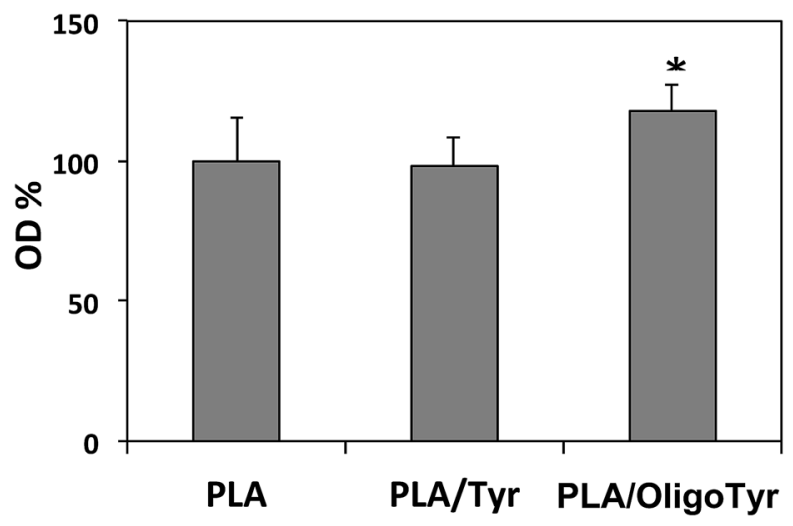

Fig. 5 ALP activities measured in the incubation medium of SaOS-2 cells after 7 days growth on pulverized PLA and the composites. Data are expressed as mean \pm SD $(n=8-16)$ of $p$-nitrophenol absorbance at $405 \mathrm{~nm}$, standardized as percent of PLA. ${ }^{*} p<0.01$ with respect to $\mathrm{PLA}$, according to multiple $t$ test with Bonferroni correction. PLA/Tyr $=$ PLA + 5\% tyrosol; PLA/OligoTyr $=$ PLA + 5\% OligoTyr.

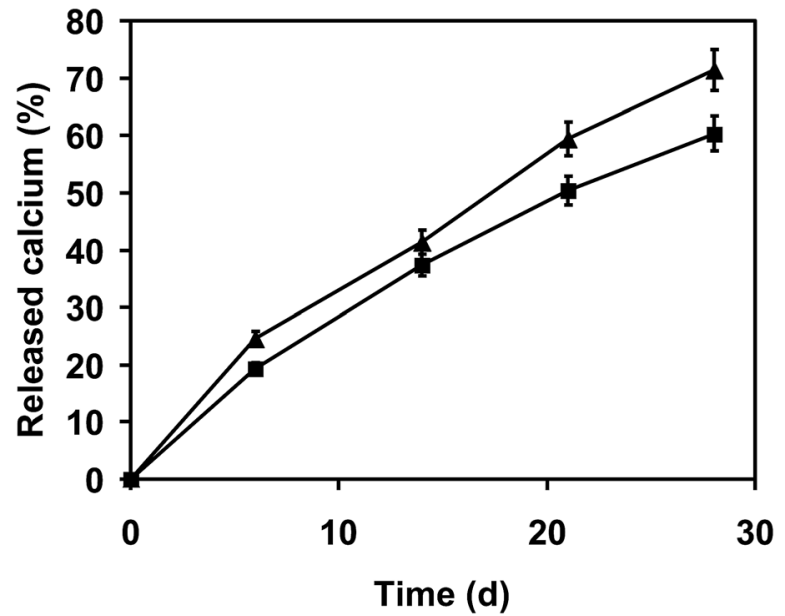

Fig. 6 Release of calcium from pure PLA (squares) and PLA containing $5 \%$ OligoTyr (triangles) scaffolds.

5\% OligoTyr not only does not interfere with calcium release from PLA scaffolds, but can actually induce a small increase, in line with a lack of chelating action which is critical to permit a continuous flux of cationic nutrients throughout the damaged area.

\section{Conclusions}

A novel phenolic polymer (OligoTyr) has been synthesized by biomimetic enzymatic oxidation of tyrosol and extensively characterized for its antioxidant capacity and potential applications in bone tissue engineering. OligoTyr proved more efficient than tyrosol in several antioxidant assays and showed good potential in promotion of osteogenesis as a stimulator of alkaline phosphatase (ALP) activity in cellular systems. Incorporation in suitably engineered 3D PLA scaffolds at high degree of porosity capable of controlling the release of the active polyphenol in physiological medium further adds to the potential of this approach for implementing biodegradable, bioresorbable materials for sustaining bone regeneration or as active fillings for repairing bone defects. The slow release of calcium ions from OligoTyr-loaded PLA scaffold is an additional valuable feature of this composite that may be worth exploiting for biological supplementation purposes.

\section{Acknowledgements}

This work was supported by a grant from Italian MIUR (PRIN 2010-2011, 2010PFLRJR "PROxi" project), and was carried out in the frame of the EuMelaNet program. We thank Prof. Giuseppe Di Silvestro for helpful discussion and suggestions.

\section{Notes and references}

1 D. Monti, G. Ottolina, G. Carrea and S. Riva, Chem. Rev., 2011, 111, 4111-4140.

2 S. K. Sahoo, W. Liu, L. A. Samuelson, J. Kumar and A. L. Cholli, Macromolecules, 2002, 35, 9990-9998. 
3 P. Zaragoza-Gasca, O. J. Villamizar-Galvez, R. GarciaArrazola, M. Gimeno and E. Barzana, Polym. Adv. Technol., 2010, 21, 454-456.

4 T. Oguchi, S. Tawaki, H. Uyama and S. Kobayashi, Bull. Chem. Soc. Jpn., 2000, 73, 1389-1396.

5 L. Zhang, W. Zhao, Z. Ma, G. Nie and Y. Cui, Eur. Polym. J., 2012, 48, 580-585.

6 T. Fukuoka, H. Uyama and S. Kobayashi, Macromolecules, 2003, 36, 8213-8215.

7 S. Kobayashi, R. Ikeda, H. Uyama, T. Tsujimoto, T. Fukuoka and H. Tonami, Polym. Mater. Sci. Eng., 2001, 84, 373-374.

8 K. Nazari, F. Adhami, A. Najjar-Safari, S. Salmani and A. Mahmoudi, Biochem. Biophys. Res. Commun., 2011, 410, 901-903.

9 H. Tonami, H. Uyama, S. Kobayashi and M. Kubota, Macromol. Chem. Phys., 1999, 200, 2365-2371.

10 T. Tanaka, M. Takahashi, H. Hagino, S.-I. Nudejima, H. Usui, T. Fujii and M. Taniguchi, Chem. Eng. Sci., 2009, 65, 569-573.

11 H. Uyama and S. Kobayashi, Curr. Org. Chem., 2003, 7, 13871397.

12 M. Reihmann and H. Ritter, Adv. Polym. Sci., 2006, 194, 1-49.

13 H. Uyama and S. Kobayashi, J. Mol. Catal. B: Enzym., 2002, 19-20, 117-127.

14 J. S. Dordick, M. A. Marietta and A. M. Klibanov, Biotechnol. Bioeng., 1987, 30, 31-36.

15 J. A. Akkara, K. J. Senecal and D. L. Kaplan, J. Polym. Sci., Part A: Polym. Chem., 1991, 29, 1561-1574.

16 S. Kobayashi, H. Uyama and S. Kimura, Chem. Rev., 2001, 101, 3793-3818.

17 V. Ambrogi, L. Panzella, P. Persico, P. Cerruti, C. A. Lonz, C. Carfagna, L. Verotta, E. Caneva, A. Napolitano and M. d'Ischia, Biomacromolecules, 2014, 15, 302-310.

18 K. Zheng, H. Tang, Q. Chen, L. Zhang, Y. Wu and Y. Cui, Polym. Degrad. Stab., 2015, 112, 27-34.

19 L. G. Rao, N. Kang and A. V. Rao, in Phytochemicals - A Global Perspective of Their Role in Nutrition and Health, ed. V. Rao, InTech, Rijeka, Croatia, 2012, pp. 467-486.

20 L. Šoltés, R. Mendichi, G. Kogan, J. Schiller, M. Stankovská and J. Arnhold, Biomacromolecules, 2006, 7, 659-668.

21 J. Tan, X. Xu, Z. Tong, J. Lin, Q. Yu, Y. Lin and W. Kuang, Bone Res., 2015, 3, 15003.

22 Y. F. He, Y. Ma, C. Gao, G. Y. Zhao, L. L. Zhang, G. F. Li, Y. Z. Pan, K. Li and Y. J. Xu, Biol. Trace Elem. Res., 2013, 152, 292-296.

23 Y. Hamada, H. Fujii and M. Fukagawa, Bone, 2009, 45, S35S38.

24 C. H. Chen, M. L. Ho, J. K. Chang, S. H. Hung and G. J. Wang, Osteoporosis Int., 2005, 16, 2039-2045.

25 M. S. Kamath, S. S. Ahmed, M. Dhanasekaran and S. W. Santosh, Int. J. Nanomed., 2014, 9, 183-195.

26 K. Hagiwara, T. Goto, M. Araki, H. Miyazaki and H. Hagiwara, Eur. J. Pharmacol., 2011, 662, 78-84.

27 Z. C. Xing, W. Meng, J. Yuan, S. Moon, Y. Jeong and I. K. Kang, J. Nanomater., 2012, 1-7.

28 J. Pan, R. Wu, X. Dai, Y. Yin, G. Pan, M. Meng, W. Shi and Y. Yan, Biomacromolecules, 2015, 16, 1131-1145.
29 Z. Xiang, P. Sarazin and B. D. Favis, Biomacromolecules, 2009, 10, 2053-2066.

30 H. Hyun, Y. H. Kim, I. B. Song, J. W. Lee, M. S. Kim, G. Khang, K. Park and H. B. Lee, Biomacromolecules, 2007, 8, 1093-1100.

31 G. Maglio, F. Nicodemi, C. Conte, R. Palumbo, P. Tirino, E. Panza, A. Ianaro, F. Ungaro and F. Quaglia, Biomacromolecules, 2011, 12, 4221-4229.

32 Y. Zhao, J. Fu, D. K. P. Ng and C. Wu, Macromol. Biosci., 2004, 4, 901-906.

33 S. Kedong, J. Lili, Z. Jingying, W. Hai, J. Zeren, M. Lim, F. Xiaoyan and L. Tianqing, Nanomaterials, 2015, 5, 415-424.

34 J. M. Raquez, Y. Habibi, M. Murariu and P. Dubois, Prog. Polym. Sci., 2013, 38, 1504-1542.

35 S. Buddhiranon, L. A. DeFine, T. S. Alexander and T. Kyu, Biomacromolecules, 2013, 14, 1423-1433.

36 P. Chuysinuan, T. Pavasant and P. Supaphol, ACS Appl. Mater. Interfaces, 2012, 4, 3031-3040.

37 W. Gou, X. Wang, J. Peng, Q. Lu, Y. Wang, A. Wang, Q. Guo, X. Gao, W. Xu and S. Lu, PLoS One, 2014, 9, e91317.

38 J. Jensen, D. C. Kraft, H. Lysdahl, C. B. Foldager, M. Chen, A. A. Kristiansen, J. H. Rölfing and C. E. Bünger, Tissue Eng., Part A, 2015, 21, 729-739.

39 J. Hong, L. M. Alvarez, N. J. Shah, L. G. Griffith, B. S. Kim, K. Char and P. T. Hammond, Drug Delivery Transl. Res., 2012, 2, 375-383.

40 A. M. Puga, A. Rey-Rico, B. Magariños, C. Alvarez-Lorenzo and A. Concheiro, Acta Biomater., 2012, 8, 1507-1518.

41 Y. Ji, L. Wang, D. C. Watts, H. Qiu, T. You, F. Deng and X. Wu, Dent. Mater., 2014, 30, 1263-1273.

42 M. G. Yeo, W. K. Jung and G. H. Kim, J. Mater. Chem., 2012, 22, 3568-3577.

43 A. Napolitano, M. de Lucia, L. Panzella and M. d'Ischia, in Olives and Olive Oil In Health and Disease Prevention, ed. V. R. Preedy and R. R. Watson, Academic Press, Oxford, 2010, pp. 1225-1232.

44 E. Fragopoulou, T. Nomikos, H. C. Karantonis, C. Apostolakis, E. Pliakis, M. Samiotaki, G. Panayotou and S. Antonopoulou, J. Agric. Food Chem., 2007, 55, 80-89.

45 I. G. Je, D. S. Kim, S. W. Kim, S. Lee, H. S. Lee, E. K. Park, D. Khang and S. H. Kim, PLoS One, 2015, 10, e0129829.

46 S. D. Sommerfeld, Z. Zhang, M. C. Costache, S. L. Vega and J. Kohn, Biomacromolecules, 2014, 15, 830-836.

47 I. C. Vlachogianni, E. Fragopoulou, I. K. Kostakis and S. Antonopoulou, Food Chem., 2015, 177, 165-173.

48 A. Napolitano, L. Panzella, M. Savarese, R. Sacchi, I. Giudicianni, L. Paolillo and M. d'Ischia, Chem. Res. Toxicol., 2004, 17, 1329-1337.

49 P. Goupy, C. Dufour, M. Loonis and O. Dangles, J. Agric. Food Chem., 2003, 51, 615-622.

50 I. F. F. Benzie and J. J. Strain, Anal. Biochem., 1996, 239, 7076.

51 M. Ozyurek, B. Bektasoglu, K. Guclu and R. Apak, Anal. Chim. Acta, 2008, 616, 196-206.

52 M. Clericuzio, B. Burlando, G. Gandini, S. Tinello, E. Ranzato, S. Martinotti and L. Cornara, J. Nat. Med., 2014, 68, 31-37. 
53 S. Martinotti, L. Mazzucco, V. Balbo, M. Patrone, M. Mozzati, E. Ranzato and B. Burlando, Curr. Pharm. Biotechnol., 2014, 15, 120-126.

54 J. F. van Staden and D. Malan, Talanta, 1996, 43, 881-888.

55 L. Basilissi, H. Farina, G. Di Silvestro and M. A. Ortenzi, J. Appl. Polym. Sci., 2013, 128, 3057-3063.

56 J. F. Turner, A. Riga, A. O'Connor, J. Zhang and J. J. Collis, J. Therm. Anal. Calorim., 2004, 75, 257-268.

57 S. F. Mahmous and S. E. Bialkowski, Appl. Spectrosc., 1995, 49, 1669-1676.

58 C. J. Jacobs and G. S. Parks, J. Am. Chem. Soc., 1934, 56, 15131517.
59 B. V. Lebedev, I. B. Rabinovich, V. I. Milov and A. M. Sladkov, J. Chem. Thermodyn., 1978, 10, 321-329.

60 S. S. Liao, F. Z. Cui, W. Zhang and Q. L. Feng, J. Biomed. Mater. Res., Part B, 2004, 69, 158-165.

61 K. Song, L. Ji, J. Zhang, H. Wang, Z. Jiao, L. Mayasari, X. Fu and T. Liu, Nanomaterials, 2015, 5, 415-424.

62 P. A. George, K. Quinn and J. J. Cooper-White, Biomaterials, 2010, 31, 641-647.

63 A. Zhu, M. Zhang, J. Wu and J. Shen, Biomaterials, 2002, 23, 4657-4665.

64 M. Yeo, H. Lee and G. Kim, Biomacromolecules, 2011, 12, 502-510. 